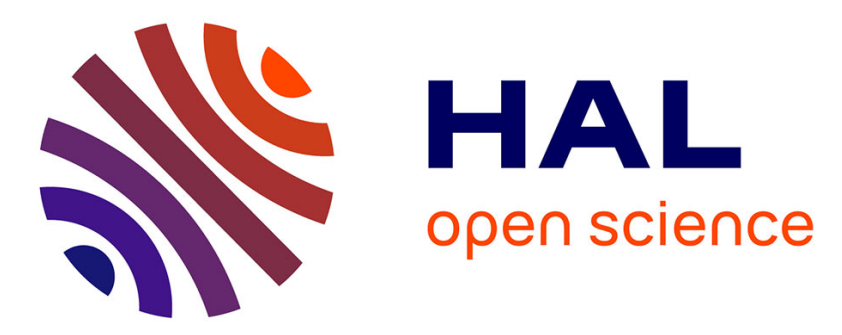

\title{
EEG $\mu$ rhythm in virtual reality reveals that motor coding of visual objects in peripersonal space is task dependent
}

\author{
Yannick Wamain, François Gabrielli, Yann Coello
}

\section{To cite this version:}

Yannick Wamain, François Gabrielli, Yann Coello. EEG $\mu$ rhythm in virtual reality reveals that motor coding of visual objects in peripersonal space is task dependent. Cortex, 2016, Cortex, 74, pp.20-30. 10.1016/j.cortex.2015.10.006 . hal-02450493

\section{HAL Id: hal-02450493 \\ https://hal.univ-lille.fr/hal-02450493}

Submitted on 9 Dec 2020

HAL is a multi-disciplinary open access archive for the deposit and dissemination of scientific research documents, whether they are published or not. The documents may come from teaching and research institutions in France or abroad, or from public or private research centers.
L'archive ouverte pluridisciplinaire HAL, est destinée au dépôt et à la diffusion de documents scientifiques de niveau recherche, publiés ou non, émanant des établissements d'enseignement et de recherche français ou étrangers, des laboratoires publics ou privés. 


\title{
EEG $\mu$ rhythm in virtual reality reveals that motor coding of visual objects in peripersonal space is task dependent
}

\author{
Yannick Wamain, François Gabrielli, Yann Coello \\ SCALab UMR CNRS 9193, Univ Lille, France
}

Running title: $\mu$ rhythm in the perception of virtual objects

Keywords: Vision, EEG, reachability, peripersonal space, $\mu$ rhythm.

\author{
Mailing Address: \\ Pr. Yann Coello \\ SCALab UMR CNRS 9193 \\ Université Charles de Gaulle-Lille3 \\ B.P. 60149 \\ 59653 Villeneuve d'Ascq Cedex, France
}

Tel: +33-3-20-41-64-46

Fax: +33-3-20-41-60-32

Email: yann.coello@univ-lille3.fr 


\begin{abstract}
Previous fMRI studies have shown that the visual perception of manipulable objects spontaneously involves the sensorimotor system, especially when the objects are located in peripersonal space. However, it has also been suggested that the motor coding of manipulable objects perceived in peripersonal space depends on an anticipation to interact with them. The present study aims at clarifying this issue by analyzing healthy adults' EEG activity on the centro-parietal region while perceptually judging intrinsic (prototypical or distorted shape) or extrinsic (reachable or not reachable location) properties of visual objects. In both the object identification and reachability judgment tasks, time-frequency decomposition of EEG signals was performed across the first $1000 \mathrm{~ms}$ following object presentation for trials on which no poststimulus response was required (90\% of the trials). Event-Related-(De)Synchronization (ERD/S) of $\mu$ rhythm was computed using the $150 \mathrm{~ms}$ pre-stimulus period as baseline. In the reachability judgment task, EEG analysis showed a desynchronization of $\mu$ rhythm starting $300 \mathrm{~ms}$ after object presentation, but only when the objects were presented with a prototypical shape in peripersonal space. For those objects, desynchronization of $\mu$ rhythm diminished progressively from peripersonal to extrapersonal space. By contrast, no such gradient was observed in the object identification task. On the whole, these data indicate that motor coding of visual objects expressed in the $\mu$ rhythm depends on an object's shape and location in space, but also on the goal of the perceptual task.
\end{abstract}

Keywords: Vision, EEG, reachability, peripersonal space, $\mu$ rhythm. 


\section{Introduction}

Visual space is not a continuum. Rather, it has a series of perceptual thresholds delimitating external environment in functional sub-spaces purposefully linked to behavior. The functional subdivision of visual space was suggested early by ethologists like Hediger (1934), who developed in the mid-twentieth century the idea that the spatial structure of the environment is perceived by most animal species through thresholds delimitating embedded zones of safety surrounding their body and used to control flight or fight. It was also shown that the size of the safety zones is mainly dependent on the animal's capacity to initiate fast motor reactions, which correlates with their weight (Blumstein \& Daniel, 2005). This idea of perceptual thresholds was later echoed by psychologists and cognitive neuroscientists to stipulate the fact that in humans visual information from the surroundings is processed partly as a function of behavioral purposes (Hall, 1996; Grüsser, 1983; Paillard, 1991; Previc, 1998; Vishton \& Cutting, 1995). According to Previc (1998), peripersonal space is processed differently from extrapersonal space because peripersonal space in particular contains the objects that we can immediately reach and manipulate. Moreover, peripersonal space specifies our private area in social contexts, and contains any obstacles to which we must pay attention in order to avoid collisions, in particular when gesturing (Cardellicchio, Sinigaglia, \& Costantini, 2011; Coello, Bourgeois, \& Iachini, 2012; Holmes \& Spence, 2004; Iachini, Coello, Frassinetti, \& Ruggiero, 2014).

Psychophysical and non-invasive brain imaging studies have provided evidence that manipulable objects in peripersonal space are perceived in relation to motor capacities (Delevoye-Turrell, Bartolo, \& Coello, 2010; Fischer, 2000; Heft, 1993; Mark et al., 1997; Robinovitch, 1998; Witt \& Proffitt, 2008). For instance, artificially modifying the spatial result of a manual reaching action (Bourgeois \& Coello, 2012), or extending reachable space with tools 
(Bourgeois, Farnè, \& Coello, 2014) entails subsequent modification of encoding in the peripersonal space. Additionally, Costantini, Ambrosini, Scorolli, \& Borghi (2011) showed that an object's orientation (e.g. the handle of a mug) influences the time required to initiate a remote motor action relating to that object, but only when the latter is presented in peripersonal space. In the same vein, Iachini et al. (2014) showed that the right-left localization of manipulable objects presented in peripersonal space using immersive virtual reality is facilitated when the arm can move freely compared to a condition in which the arm were stuck behind the back, creating motor interference.

Brain imaging data have also suggested that the motor system is involved in the perception of manipulable objects located in peripersonal space. For instance, Bartolo et al. (2014) found that reachability estimates trigger activation within a fronto-parietal network including the cerebellum, but only when the stimuli are presented in peripersonal space. These brain regions overlap with the one subtending the production of actual goal-directed movements (Binkofski et al., 1999; Medendorp, Buchholz, Van Der Werf, \& Leoné, 2011) and with the network involved in the perception of others' peripersonal space (Lamm, Fischer, \& Decety, 2007). Furthermore, using transcranial magnetic stimulation (TMS) at low frequency, Coello et al. (2008) found that reachability judgments are delayed when TMS is applied to the left motor cortex, an effect not observed when TMS is applied to a control site in the left temporo-occipital area (for congruent results, see also Cardellicchio et al., 2011). Corroborating these data, Bartolo et al. (2014) showed that patients with brain damage in motor regions make specifically impaired reachability estimates.

In line with the data reported thus far, several studies have suggested that manipulable objects are automatically coded in motor terms provided that they are perceived in peripersonal 
space (Ambrosini, Sinigaglia, \& Costantini, 2012; Gallese, 2007; Gallivan, Cavina-Pratesi, \& Culham, 2009). Quinlan \& Culham (2007) showed that simply observing an object in peripersonal space triggers specific brain activations within the dorsal stream of the visual system, in particular in the reach-related area of the superior parieto-occipital cortex (see also Culham, Gallivan, Cavina-Pratesi, \& Quinlan, 2008; Gallivan et al., 2009). By contrast, Witt et al. (2005) found that motor-related perception of objects in peripersonal space is altered when holding a tool, but only when one intends to use it. This suggests that the perception of objects in peripersonal space involves the motor system only when the actor anticipates interaction with the objects. However, Witt et al. did not provide brain correlates in that study, so although the data summarized above strongly suggests that objects in peripersonal space are spontaneously coded in motor terms, it has not yet been shown whether the involvement of the motor system depends on the goal of the perceptual task as understood by the actor. The present study aims to answer that question.

In the present experiment, we presented objects in either peripersonal or extrapersonal space, and orthogonal to that factor, participants performed two perceptual discrimination tasks. One of the tasks involved reachability judgments and focused on motor-related visual information (reachable versus unreachable object location). The other perceptual discrimination task involved object identification focusing on the objects' visual attributes (prototypical versus distorted object shape). In each task, we analyzed the EEG signals through a time-frequency decomposition centered on the $\mu$ rhythm ( $8 \mathrm{~Hz}$ to $12 \mathrm{~Hz}$ ). Previous studies have reported signal attenuation within this frequency range, namely an event-related desynchronization in situations associated with motor preparation and execution (Babiloni et al., 1999; Llanos, Rodriguez, Rodriguez-Sabate, Morales, \& Sabate, 2013; Salenius, Schnitzler, Salmelin, Jousmäki, \& Hari, 
1997; Salmelin \& Hari, 1994), movement observation (Cochin, Barthelemy, Roux, \& Martineau, 1999; Pineda, Allison, \& Vankov, 2000), motor imagery (Braadbaart, Williams, \& Waiter, 2013;

Hari, 2006; Muthukumaraswamy, Johnson, \& McNair, 2004) or tactile stimulation (Coll, Bird, Catmur, \& Press, 2014; Perry, Bentin, Bartal, Lamm, \& Decety, 2010). Furthermore, Proverbio (2012) showed that early desynchronization of $\mu$ rhythm is maximal over the centro-parietal region when observing manipulable objects. In the present study, within a virtual reality environment we asked whether event-related desynchronization in the $8 \mathrm{~Hz}-12 \mathrm{~Hz}$ bandwidth over the centro-parietal region is modulated by the prototypical versus distorted visual shape of reachable versus unreachable objects ${ }^{1}$. We expected desynchronization of $\mu$ rhythm to be maximal for prototypical (manipulable) objects located in peripersonal space. Furthermore, if objects in peripersonal space are automatically coded in motor terms, we expected a similar pattern of results when participants performed a reachability judgment task versus an object identification task.

\section{Methods}

Nineteen healthy adults (mean age 23.55; age range 19-29; 12 women) participated in the study. They were right-handed, according to the Edinburgh Test (handedness quotients 60\%100\%; mean 87.7\%; Oldfield, 1971) and all had normal or corrected-to-normal visual acuity. Recruitment of participants and testing conformed with the 2008 Helsinki Declaration, and the local Ethics Committee specifically approved this study.

\footnotetext{
1 Because objects were presented in virtual reality, we could display them with either their natural shape (prototypical objects) or with a distorted shape using a Gaussian scattering algorithm (distorted objects). This procedure allowed control of the amount of visual information (pixels) characterizing prototypical / distorted pairs of stimuli while substantially modifying the manipulable aspects of objects.
} 


\subsection{Visual scene and stimuli}

Images of 20 different visual objects within a structured virtual scene were generated using MatLab 6.5 (Mathworks, Natick, Mass., U.S.A.) and Psychophysics Toolbox extensions (Brainard, 1997; Kleiner, Brainard, \& Pelli, 2007; Pelli, 1997). Visual scenes were presented on a $2 \mathrm{~m}$ x $4 \mathrm{~m}$ rear projection screen using a 3D stereoscopic projector (Christie Mirage 4K35) generating images at $120 \mathrm{~Hz}$ with a $4 \mathrm{~K}$ spatial resolution (3840 x 2060 pixels). Active 3D eyewear (Christie) was used for producing 3D image perception. Stereoscopic images were displayed with off-axis projection by using non-symmetrical camera frustums in order to prevent vertical parallax while providing comfortable stereo pairs. The images were generated taking into account participants' height and inter-pupillary distance. Thus, for each stimulus two different images were computed, one for each eye, and displayed alternately at the rate of 8.33 ms per eye. Normal fusion created the illusion of viewing a single object. Relative size and perspective cues as well as binocular disparity were used to induce the 3D perception of the visual scene and objects. The visual scene (Figure 1A) consisted of a rectangular room (length 10 $\mathrm{m}$, width $7 \mathrm{~m}$, height $4 \mathrm{~m}$ ), with wooden textured roof and tile-layered textured walls and floor. There was also a wooden textured table, displayed using custom Matlab code based on Psychophysics Toolbox and OpenGL instructions. No lighting was used in generating the scene. Objects were presented either in their original shape (prototypical objects, Figure 1B), with the same dark green color, or with a distorted shape using a Gaussian scattering algorithm (distorted objects, Figure 1C). The prototypical objects were selected from public and open source 3D objects databases and converted into OpenGL-compatible format (arrays of vertices, normals and faces) for fast loading and rendering. Prototypical objects were selected so that they spontaneously evoked a power grip (e.g., bottle, glass - see the complete list of stimuli, 
Appendix). Vertices arrays were scaled to a normalized width of $7.5 \mathrm{~cm}$ diameter at the most graspable section of the object. Objects were presented on the table, with light shading to enhance 3D perception. Distorted versions of these objects were obtained by shifting pairs of pixels horizontally: firstly, the object was detected in the picture and framed by a rectangle. Then, each pixel within the rectangle was exchanged with another pixel on the same horizontal line, placed at a distance randomly calculated from a Gaussian distribution with a mean centered on the pixel and a standard deviation of 30 pixels. This process maintains the color and light flow of the visual stimulus while significantly altering its visual features. The distortion was thus associated with a reduction of the alignment of contour items, making the shape of the object less salient. Each prototypical object was thus associated with a distorted object within a picture that contained the same pixels, thanks to the pair-switching process, but minus the global shape and manipulable appearance.

$<$ Insert Figure 1 about here $>$

Prototypical and distorted objects were presented at various distances according to the participant's vantage point. For each participant distances were individually normalized as a function of the actual maximum reachable distance as determined in a pre-experiment session. Prototypical and distorted objects were presented at $-50 \%,-60 \%,-70 \%$ (peripersonal space), $-10 \%, 0 \%,+10 \%$ (boundary of peripersonal space) $,+50 \%,+60 \%,+70 \%$ (extrapersonal space) of actual maximum reachable distance.

\subsection{Procedure}

Before starting the experiment, the experimenter explained the virtual environment and the EEG equipment, as well as the overall goal of the study. The participant was then seated 
facing the screen in a dimly illuminated room and worn the Active 3D eyewear system. Two response pedals were placed under the participant's feet and were connected to the computer in order to record the participant's responses during the perceptual tasks (Figure 1A). Participants were informed that they were to perform two distinct tasks (counterbalanced block sessions): an Object Identification task (OI) and a Reachability Judgment task (RJ). In the OI task, participants were to judge whether the stimulus was a distorted or a prototypical object. In the RJ task, they were to judge if the stimulus was reachable or not.

\section{Determination of individual peripersonal space}

In the pre-experiment session, cylindrical objects were presented in the stereoscopic virtual environment and the participant's task was to judge whether the cylindrical object $(7 \mathrm{~cm} \mathrm{x}$ $7.5 \mathrm{~cm}$, prototypical shape) presented at different distances was fully graspable with the right hand or not (no actual movements were performed). The virtual environment was similar to the one used in the experiment except the cylindrical objects were used only in the pre-experiment session. Participants' responses were provided using the right and left pedals (for reachable and unreachable responses, counterbalanced across participants). Pedals were used for responding to avoid interaction with the reachability judgment task performed according to what is reachable with the right hand. Each stimulus remained visible on the screen until the response was provided. The distance between the cylinder and the participant varied randomly between $20 \mathrm{~cm}$ and $160 \mathrm{~cm}$ in steps of $5 \mathrm{~cm}$ ( 29 distances), and each position was repeated 4 times thus yielding a total of 116 trials. This pre-experiment session was used to determine the extent of peripersonal space for each participant. It was also used to check whether the participant accurately perceived the 3D stimuli through stereoscopic vision and without visual discomfort (verbal report). The 
boundary of peripersonal space was determined using a maximum likelihood fit based on the second-order derivatives (quasi-newton method) to obtain the logit regression model that best fitted the participant's reachable / unreachable responses using the equation: $y=e^{(\alpha+\beta X)} /(1+$ $\left.\mathrm{e}^{(\alpha+\beta \mathrm{X})}\right)$, in which $\mathrm{y}$ was the participant's response, $\mathrm{X}$ was the distance of the stimulus, and ($\alpha / \beta)$ was the critical value of $X$ corresponding to the transition between reachable and unreachable stimuli, thus expressing the perceived maximum reachable distance. Individual boundaries of peripersonal space were used to select the stimuli presented in the experimental tasks. During the pre-experiment session, we also measured the length of the participant's right arm, i.e., the distance from the acromion to the tip of index finger.

\section{Experimental tasks}

In the OI and RJ tasks (task order counterbalanced across participants) prototypical and distorted objects were presented at different distances for $1000 \mathrm{~ms}$ with inter-stimuli-interval (ISI) randomly varied between $1500 \mathrm{~ms}$ and $1900 \mathrm{~ms}$. In both tasks, the combination of type of object (prototypical/distorted) and its distance from the participant ( 9 different distances) was randomly selected in each trial. In $90 \%$ of the trials, the ISI was a blank period. In $10 \%$ of the trials, a question mark picture was presented for $1000 \mathrm{~ms}$ after the stimulus disappeared indicating to the participants that they had to provide their response by pressing one of the pedals, but in relation to different visual attributes depending on the task. In the OI task, the participant was to judge if the object presented was a prototypical or a distorted object. In the RJ task, whether the object was reachable or not reachable (i.e., whether the presented object could be fully grasped or not). In the two tasks, association between foot and response was counterbalanced across participants. Participants then performed 396 trials [2 Object types 
(prototypical/distorted) $\times 3$ Spaces (peripersonal, extrapersonal, boundary of peripersonal space) $\times 3$ Distances $\times 20$ Objects +36 trials requiring a response $(10 \%)$ ]. Trials triggering a response were randomly presented but assigned according to a balanced combination of objects, distances, and visual shape. The experimental session lasted about 25 minutes, including breaks. Only the $90 \%$ of trials without behavioral responses were used for EEG analyses.

\subsection{EEG acquisition and processing}

Participants were equipped with an EEG Active Two Biosemi system (Amsterdam, the Netherlands) comprising 128 active electrodes mounted on a cap (10-20 International system Electro-Cap Inc). EEG signal was continuously recorded at $1024 \mathrm{~Hz}$ throughout the experiment, and electrode offset was kept below $20 \mathrm{mV}$. In order to monitor eye movements and blinks, two additional electrodes were placed at lateral canthi and below the eyes. For offline analysis, continuous EEG signal was filtered $(1-100 \mathrm{~Hz})$ and recalculated based on average reference using EEGLAB software (Delorme \& Makeig, 2004), after periods with excessive noise artifacts were removed. ICA-based artifact correction was used in order to correct for blink artifacts (Delorme, Sejnowski, \& Makeig, 2007). The signal was then divided into periods of $1400 \mathrm{~ms}$ around the onset of the target object (200 ms pre-target and $1200 \mathrm{~ms}$ post target onset). Epochs still contaminated by muscular contractions or excessive deflection $( \pm 75 \mu \mathrm{V})$ were detected by visual inspection of the data and were excluded (total rejection rate was about 5\%). At this step, two participants were removed from the analysis because they presented persistent excessive muscle artifacts. Event-related changes in the oscillatory activity were quantified using a timefrequency wavelet decomposition of the continuous EEG signals between 1 and $45 \mathrm{~Hz}$ (complex Morlet's wavelets, ratio fo/ $/ \mathrm{f}=7$ ) implemented in a Matlab toolbox (Fieldtrip software; for a 
complete description of this method see Tallon-Baudry, Bertrand, Delpuech, \& Pernier, 1996). In order to represent frequency modulation induced by object presentation, the mean spectral power of the pre-event period (from $-150 \mathrm{~ms}$ to $0 \mathrm{~ms}$ ) was considered to be a baseline level and was subtracted from each time point for a given frequency and participant for the next $1000 \mathrm{~ms}$. Finally, the data were expressed as \%-of-baseline by dividing the results of each subtraction by the baseline value. For each participant, mean power of $\mu$ rhythm was quantified $(8 \mathrm{~Hz}-12 \mathrm{~Hz})$ on the centro-parietal site corresponding to electrodes A1, A2, A3, B1, B2, D15, D16 (Behmer \& Jantzen, 2011; Nyström, Ljunghammar, Rosander, \& Hofsten, 2011; Perry \& Bentin, 2009, 2011; Pfurtscheller, Brunner, Schlögl, \& Lopes da Silva, 2006; Pineda, Giromini, Porcelli, Parolin, \& Viglione, 2011; Proverbio, 2012). To quantify the change induced by stimulus presentation per se in the two tasks, we averaged the $\mu$ change across the entire stimulus presentation period (time-window 0-1000 ms).

\subsection{EEG data analysis}

EEG data were analyzed for each participant in the two perceptual tasks, pooling the data across the 20 objects. Thus, a 2 Tasks (OI, RJ) x 2 Object types (prototypical, distorted) x 3 Spaces (peripersonal, extrapersonal, boundary of peripersonal space) analysis of variance (ANOVA) was computed on the mean $\mu$ power change. In view of previous findings (Cardellicchio et al., 2011; Costantini et al., 2011; Proverbio, 2012), we predicted that not only the objects' shape but also their location in space would affect EEG activity over the centroparietal region. In particular, a $\mu$ power change gradient was expected going from objects in peripersonal space to objects in the boundary to those in extrapersonal space. Such a gradient should however be more prominent for prototypical (manipulable) than for distorted (non- 
manipulable) objects, and foremost in the RJ task. These hypotheses were tested through specific contrasts, and the significance of the mean $\mu$ power change in each condition was tested using a one-sample t-test with $\mathrm{H}_{\mathrm{o}}=0$. Other effects were tested through post-hoc comparisons using Bonferroni correction to maintain $\alpha=5 \%$ across multiple comparisons.

Finally, to account for the time-scale of $\mu$ power change as a function of object distance, we compared $\mu$ power change induced by visual presentation of objects located in peripersonal versus extrapersonal space at each time bin using t-test comparisons. This procedure was performed for prototypical as well as distorted objects in each task.

\section{Results}

\subsection{Pre-experiment determination of peripersonal space}

In the pre-experiment task performed in order to measure individual peripersonal space in the virtual environment, we found that the boundary of peripersonal space was located on average $87.5 \mathrm{~cm}$ (range 43-122; SD: $24.3 \mathrm{~cm}$ ) from the body. This distance corresponded to an overestimation of actual reachable capacities (15.03\%) when considering mean arm length (73 $\mathrm{cm}$, range $65-80 \mathrm{~cm})$. Moreover, ANOVA performed on response time revealed a main effect of object location $\left(\mathrm{F}_{2,22}=35.2, \mathrm{p}=0.001, \eta_{\mathrm{p}}{ }_{\mathrm{p}}=.69\right)$. T-test comparisons indicated that participants took longer to respond in the RJ task when objects were located at the boundary of peripersonal space (mean: $1173 \mathrm{~ms}$; SD: $173 \mathrm{~ms})$ than in peripersonal $\left(915 \mathrm{~ms}\right.$; SD: $191 \mathrm{~ms}$; $\mathrm{t}_{16}=5.04, \mathrm{p}=$ 0.001) or extrapersonal (mean: $871 \mathrm{~ms}$; $\mathrm{SD}: 121 \mathrm{~ms} ; \mathrm{t}_{16}=10.14, \mathrm{p}=0.001$ ) space. No difference was observed in time to respond when comparing objects presented either in peripersonal or extrapersonal space $\left(\mathrm{t}_{16}=0.53, \mathrm{p}=0.30\right)$. 


\subsection{Analysis of EEG $\mu$ rhythm $(8 \mathrm{~Hz}-12 \mathrm{~Hz})$}

Time-frequency analysis revealed specific patterns of results depending on the task, the object location and its visual shape (Figure 2).

$<$ Insert Figure 2 about here $>$

$\mu$ power change on the $0-1000 \mathrm{~ms}$ time-window was analyzed according to Object type and Space in the RJ and OI tasks. The ANOVA performed on the average $\mu$ power change did not reveal a reliable effect of Space or Task $\left(F_{1,16}=0.942, p=0.35\right.$ and $\left.F_{1,16}=2.12, p=0.13\right)$, but there was a marginal effect of Object type $\left(\mathrm{F}_{1,16}=4.44, \mathrm{p}=0.05\right)$. The Task $\times$ Object type, Task $\times$ Space or Object type $\times$ Space interactions were all nonsignificant for $\mu$ power change (respectively, $\mathrm{F}_{2,32}=2.93, \mathrm{p}=0.11 ; \mathrm{F}_{2,32}=0.74, \mathrm{p}=0.48$ and $\mathrm{F}_{2,32}=2.06, \mathrm{p}=0.14$ ).

The ANOVA performed on the average $\mu$ value revealed a significant Task $\times$ Object type $\times$ Space interaction $\left(\mathrm{F}_{2,32}=4.22, \mathrm{p}=0.03, \eta_{\mathrm{p}}^{2}=0.19\right)$. As shown in Figure 3, planned comparisons revealed that $\mu$ power change induced by the presentation of prototypical objects in peripersonal space was significantly reduced compared to that observed for the same objects in extrapersonal space $\left(F_{1,16}=8.26, p=0.01\right)$, although only in the RJ task $\left(F_{1,16}=0.15, p=0.70\right.$ for OI task $)$. Furthermore, $\mu$ power change induced by objects located at the boundary of peripersonal space did not differ from the average of $\mu$ power change induced by objects presented in either the peripersonal or the extrapersonal space (respectively, $\mathrm{F}_{1,16}=0.18, \mathrm{p}=0.67$ and $\mathrm{F}_{1,16}=1.00, \mathrm{p}=$ 0.33 for RJ and OI task).

Comparison to 0 indicated that in the RJ task, $\mu$ power change reached significance for prototypical objects located in peripersonal space $\left(\mathrm{t}_{16}=2.17, \mathrm{p}=0.04\right)$ but not for those located at the boundary of peripersonal or in extrapersonal space (respectively, $\mathrm{t}_{16}=1.31, \mathrm{p}=0.21$ and $\left.\mathrm{t}_{16}=0.30, \mathrm{p}=0.76\right)$. No significant $\mu$ power change was observed for distorted objects in the RJ 
$\operatorname{task}\left(\mathrm{t}_{16}=1.30, \mathrm{p}=0.21 ; \mathrm{t}_{16}=1.22, \mathrm{p}=0.24\right.$ and $\mathrm{t}_{16}=1.71, \mathrm{p}=0.10$ for peripersonal, boundary and extrapersonal space, respectively).

Taking together, we observed a $\mu$ power change gradient when the participant judged the reachability of prototypical objects, whereas no similar pattern of results appeared for distorted objects. Indeed, prototypical objects in peripersonal space yielded maximal negative $\mu$ power change whereas the same objects in extrapersonal space yielded minimal negative $\mu$ power change. Prototypical objects located at the boundary of peripersonal space yielded an intermediary pattern of results.

By contrast with these results, $\mu$ power change was observed neither for distorted objects in the RJ task, nor for prototypical or distorted objects in the OI task. Indeed, none of the linear contrasts (respectively, $F_{1,16}=0.88, p=0.36 ; F_{1,16}=0.41, p=0.53$ and $F_{1,16}=0.15, p=0.70$ for distorted objects in the RJ and OI tasks and prototypical objects in the OI task) or quadratic contrasts (respectively, $F_{1,16}=0.21, p=0.65 ; F_{1,16}=2.10, p=0.16$ and $F_{1,16}=1.00, p=0.33$ for distorted objects in the RJ and OI tasks and prototypical objects in the OI task) were significant. Moreover, comparison to 0 in the OI task indicated that $\mu$ power change never reached significance when considering either the prototypical objects $\left(\mathrm{t}_{16}=0.81, \mathrm{p}=0.42 ; \mathrm{t}_{16}=0.01, \mathrm{p}=\right.$ 0.99 and $\mathrm{t}_{16}=0.54, \mathrm{p}=0.59$ for peripersonal, boundary and extrapersonal space respectively) or the distorted objects $\left(\mathrm{t}_{16}=1.32, \mathrm{p}=0.20 ; \mathrm{t}_{16}=2.19, \mathrm{p}=0.09\right.$ for boundary and extrapersonal space respectively) except in the peripersonal space $\left(\mathrm{t}_{16}=2.17, \mathrm{p}=0.04\right)$.

The difference in $\mu$ power change induced by prototypical objects versus distorted objects reached significance in peripersonal space and extrapersonal for the RJ task (respectively, $\mathrm{F}_{1,16}=7.00, \mathrm{p}=0.02$ and $\mathrm{F}_{1,16}=6.71, \mathrm{p}=0.02$ ), while no difference appeared at the boundary of reachable space $\left(\mathrm{F}_{1,16}=0.23, \mathrm{p}=0.63\right)$. Similar comparisons for the OI task did not 
reveal reliable differences $\left(F_{1,16}=3.20, p=0.09 ; F_{1,16}=3.90, p=0.07\right.$ and $F_{1,16}=2.91, p=0.11$ in peripersonal, boundary or extrapersonal space, respectively).

$$
<\text { Insert Figure } 3 \text { about here }>
$$

Finally, as shown in Figure 4, we used t-tests to compare the moment in time in the RJ task when $\mu$ power changed significantly for objects located in peripersonal space versus extrapersonal space (the 3 object locations being averaged in each space). This comparison indicated that, for prototypical objects, a significant difference appeared $300 \mathrm{~ms}$ following object presentation, and the difference remained significant to the end of the presentation period. No such effect was observed for distorted objects.

$$
<\text { Insert Figure } 4 \text { about here }>
$$

\section{Discussion}

The aim of the present study was to investigate in a visual discrimination task, focusing on either intrinsic features of objects (prototypical or distorted shape) or extrinsic features (reachable or not reachable location), whether the involvement of the motor neural network depends on the shape of visual objects, their location in space or the goal of the perceptual task as understood by the participant. Specifically, we studied the temporal dynamic of the activation of the motor neural network using a novel experimental design combining 3D virtual reality with the recording of brain signals from EEG. Whatever the perceptual discrimination task reachability or identification, this design allowed us to quantify the modulation of $\mu$ rhythm (8$12 \mathrm{~Hz}$ ) over the centro-parietal region and to analyze its time course when prototypical 
(manipulable) or distorted (non-manipulable) objects were presented at different locations in space.

Analyzing EEG signals over the centro-parietal region revealed that $\mu$ power change induced by the presentation of visual objects was sensitive to three factors: object features (prototypical or distorted shape), object location (peripersonal, extrapersonal, or boundary of peripersonal space) and also the goal of the perceptual discrimination task (reachability judgment or object identification task). On the whole, the greatest $\mu$ desynchronization was observed when participants judged the reachability of prototypical objects presented in peripersonal space. Such desynchronization reduced progressively when the objects approached extrapersonal space. By contrast, no such gradient was observed for similar objects when participants were performing the object identification task - which thus stood as an effective control procedure. Thus, differences in the $\mu$ wave depending on objects' features and their location in space suggest that manipulable objects located in peripersonal space are coded in a specific way, one that is different from the perceptual coding of visual objects in extrapersonal space. However, the effect of the objects' shape and location was modulated by the goal of the perceptual discrimination task - identification vs. reachability.

EEG signal desynchronization is a reliable correlate of excited neural networks or activated cortical areas (Goldman, Stern, Engel, \& Cohen, 2002). One interpretation for the observed pattern of $\mu$ desynchronization depending on the location of manipulable objects could be that the modulation of $\mu$ rhythm reflects low-level visual processing of the stimuli, which varied in size as well as in luminosity and colour contrast, when receding in space. However, the fact that distorted non-manipulable objects did not produce the same desynchronization of $\mu$ rhythm, even when located in peripersonal space, led us to reject this interpretation. Furthermore, 
$\mu$ rhythm attenuation has been found to be minimally affected by visual stimulation alone (Pineda, 2005). An alternative interpretation of the pattern of $\mu$ desynchronization observed in the present study is that modulation of $\mu$ rhythm reflects sensorimotor processing in the frontoparietal networks (Cochin et al., 1999; Ciganek, 1959; Jasper \& Penfield, 1949; Kuhlman, 1978; Gert Pfurtscheller \& Neuper, 1994; Proverbio, 2012; Pfurtscheller et al., 1996). Previous studies have indeed revealed that $\mu$ rhythm is modified by voluntary motor action, whether actually performed, mentally simulated or observed being performed by coparticipants (Babiloni et al., 1999; Braadbaart et al., 2013; Cochin et al., 1999; Hari, 2006; Llanos et al., 2013; Muthukumaraswamy et al., 2004; Muthukumaraswamy \& Johnson, 2004; Pfurtscheller et al., 2006; Salenius et al., 1997; Salmelin \& Hari, 1994). Moreover, passive observation of manipulable objects not only recruits a neural motor network overlapping with that activated during object-oriented motor productions as revealed by fMRI studies (Chao \& Martin, 2000; Gallivan, McLean, \& Culham, 2011; Grafton \& Hamilton, 2007; Grèzes \& Decety, 2002; Quinlan \& Culham, 2007), but also produces a comparable modulation of the $\mu$ rhythm (8-12 $\mathrm{Hz}$ ) over the centro-parietal region (Proverbio, 2012). Thus, even in the absence of obvious intention to perform an overt motor action, the neural motor network activated during the perception of visual objects seems to depend on their appearance as manipulable or not. The new finding in the present study is that desynchronization of $\mu$ rhythm associated with the motor coding of visual objects depends also on the their location in peripersonal space.

This interpretation agrees with Culham et al. (2008), whose fMRI study showed that observing manipulable objects triggers specific brain activations within the dorsal stream of the visual system, in particular in the reach-related area of the superior parieto-occipital cortexSPOC (see also Gallivan et al., 2009, 2011; Quinlan \& Culham, 2007). Interestingly, this motor- 
related activation when observing manipulable objects was essentially observed when objects were located in the range of space reachable with the dominant hand, even in the absence of an intention to act. The interpretation of these data was that SPOC is particularly responsive to stimuli presented within reach in relation to upper-limb movements. No similar activation was observed for objects located at the same distance but reachable only with the non-dominant hand. These observations suggested that among object features reactivated during object perception, the observers mobilized motor knowledge and particularly their typical workspace coded as a function of their action possibilities/preferences. Corroborating these findings, Cardellichio (2011) found higher motor evoked potentials during the observation of graspable objects falling within peripersonal space compared to the observation of either non-graspable objects or graspable objects falling outside peripersonal space. Our findings corroborate and extend these observations by showing that modulation of the $\mu$ rhythm desynchronization over the centroparietal region is related to objects encoded at the neural level as a function of the observer's action capabilities. Interestingly, investigation of the temporal dynamic of $\mu$ change as a function of object location reveals that the distinction in the $\mu$ change for objects located in peripersonal and extrapersonal space appears as early as $300 \mathrm{~ms}$ following object presentation. Considering that reachability responses took on average $987 \mathrm{~ms}$ (in our paradigm, see pre-experiment), this indicates that the activation of the motor neural network arises during reachability decision making. Using a task consisting simply in observing manipulable/non-manipulable objects, Proverbio et al. (2012) found a desynchronization of $\mu$ rhythm occurring around 145-200 ms following object presentation, which is much earlier that the $300 \mathrm{~ms}$ observed in the present study. This difference might suggest different stages in the involvement of the motor neural network during object perception, depending on the visual motor properties emphasized by the 
task. Indeed, everything happens as though the motor neural network were initially responding to object affordance, and then anticipating the consequences of acting on the object in a particular context, for which more elaborated motor simulation would be necessary.

The fact that motor-related activation when processing visual objects was observed only when performing the reachability judgment task, suggests that the involvement of the motor network in the processing of visual objects in peripersonal space is not automatic but rather depends on the goal of the perceptual task. Indeed, when performing the object identification task, participants showed no modulation of the $\mu$ rhythm, even for manipulable objects presented in peripersonal space. This task-dependent modulation of $\mu$ rhythm is compatible with Witt and colleagues' recent proposal that motor coding of perceptual content relies on participants' intention or preparation to act (Witt, Proffitt, \& Epstein, 2004; Witt et al., 2005; Witt, 2011). Indeed, when the goal of our perceptual task was not object discrimination but rather reachability judgment, it required a shift of attention from visual to action-related features of the presented objects. While remaining aware that the issue of the similarity of brain areas recruited during action implementation versus perceptual processing remains to be properly addressed, we see in the results of the present study direct support for the idea that the motor neural activity that subtends the perception of visual objects located in peripersonal space can be modified by changing the goal of the perceptual task.

In conclusion, the present study underlines that virtual reality represents a relevant tool for investigating neural substrates of human behavior in situations close to real ones. Behavioral data are indeed very consistent with the previous observations made in natural contexts, in particular the slight overestimation associated with reachability estimates (Carello, Grosofsky, 
Reichel, Solomon, \& Turvey, 1989; Coello \& Iwanow, 2006; Fischer, 2000; Gabbard, Ammar, \& Lee, 2006; Rochat \& Wraga, 1997). Furthermore, using an original combination of virtual reality and EEG recording, we confirmed that manipulable objects are coded in motor terms in a perceptual task requiring no actual physical interactions with them (Coello \& Delevoye-Turrell, 2007; Culham et al., 2008; Gallese, 2009; Jeannerod, 2001). However, the new findings in the

present study are twofold. On the one hand, analysis of $\mu$ rhythm desynchronization highlighted that the motor coding of visual objects depends not only on their perceived intrinsic manipulability, but also on their location in space. On the other hand, we found that $\mu$ rhythm desynchronization depends on the goal of the perceptual task, stressing that the motor coding of manipulable objects in peripersonal space does not rely exclusively on automatic processes. Taken together, these results are important, for they reveal that the motor coding of visual objects in a perceptual task is a function not only of the objects' intrinsic and extrinsic features, but also the purpose of the sensory processing itself.

\section{Acknowledgments}

This work was funded by the French National Research Agency (ANR-11-EQPX-0023) and also supported by European funds through the program FEDER SCV-IrDIVE. The authors are grateful to John M. Belmont, Ph.D., for his helpful comments on the manuscript.

\section{References}

Ambrosini, E., Sinigaglia, C., \& Costantini, M. (2012). Tie my hands, tie my eyes. Journal of Experimental Psychology. Human Perception and Performance, 38(2), 263-6. 
Babiloni, C., Carducci, F., Cincotti, F., Rossini, P. M., Neuper, C., Pfurtscheller, G., \& Babiloni, F. (1999). Human movement-related potentials vs desynchronization of EEG alpha rhythm: a high-resolution EEG study. NeuroImage, 10(6), 658-65.

Bartolo, A., Coello, Y., Edwards, M. G., Delepoulle, S., Endo, S., \& Wing, A. M. (2014). Contribution of the motor system to the perception of reachable space: an fMRI study. The European Journal of Neuroscience, 40(12), 3807-17.

Behmer, L. P., \& Jantzen, K. J. (2011). Reading sheet music facilitates sensorimotor mudesynchronization in musicians. Clinical Neurophysiology: Official Journal of the International Federation of Clinical Neurophysiology, 122(7), 1342-7.

Binkofski, F., Buccino, G., Posse, S., Seitz, R. J., Rizzolatti, G., \& Freund, H.-J. (1999). A fronto-parietal circuit for object manipulation in man: evidence from an fMRI-study. European Journal of Neuroscience, 11(9), 3276-3286.

Blumstein, D. T., \& Daniel, J. C. (2005). The loss of anti-predator behaviour following isolation on islands. Proceedings. Biological Sciences / The Royal Society, 272(1573), 1663-8.

Bourgeois, J., \& Coello, Y. (2012). Effect of visuomotor calibration and uncertainty on the perception of peripersonal space. Attention, Perception \& Psychophysics, 74(6), 1268-83.

Bourgeois, J., Farnè, A., \& Coello, Y. (2014). Costs and benefits of tool-use on the perception of reachable space. Acta Psychologica, 148, 91-5.

Braadbaart, L., Williams, J. H. G., \& Waiter, G. D. (2013). Do mirror neuron areas mediate mu rhythm suppression during imitation and action observation? International Journal of Psychophysiology: Official Journal of the International Organization of Psychophysiology, 89(1), 99-105.

Brainard, D. H. (1997). The Psychophysics Toolbox. Spatial Vision, 10(4), 433-6. 
Cardellicchio, P., Sinigaglia, C., \& Costantini, M. (2011). The space of affordances: a TMS study. Neuropsychologia, 49(5), 1369-72.

Carello, C., Grosofsky, A., Reichel, F. D., Solomon, H. Y., \& Turvey, M. T. (1989). Visually Perceiving What is Reachable. Ecological Psychology, 1, 27-54.

Chao, L. L., \& Martin, A. (2000). Representation of Manipulable Man-Made Objects in the Dorsal Stream. NeuroImage, 12(4), 478-484.

Cochin, S., Barthelemy, C., Roux, S., \& Martineau, J. (1999). Observation and execution of movement: similarities demonstrated by quantified electroencephalography. European Journal of Neuroscience, 11(5), 1839-1842.

Coello, Y., Bartolo, A., Amiri, B., Devanne, H., Houdayer, E., \& Derambure, P. (2008). Perceiving what is reachable depends on motor representations: evidence from a transcranial magnetic stimulation study. PloS One, 3(8), e2862.

Coello, Y., Bourgeois, J., \& Iachini, T. (2012). Embodied perception of reachable space: how do we manage threatening objects? Cognitive Processing, 13 Suppl 1, S131-5.

Coello, Y., \& Delevoye-Turrell, Y. (2007). Embodiment, spatial categorisation and action. Consciousness and Cognition, 16(3), 667-83.

Coello, Y., \& Iwanow, O. (2006). Effect of structuring the workspace on cognitive and sensorimotor distance estimation: no dissociation between perception and action. Perception \& Psychophysics, 68(2), 278-89.

Coll, M.-P., Bird, G., Catmur, C., \& Press, C. (2014). Cross-modal repetition effects in the mu rhythm indicate tactile mirroring during action observation. Cortex; a Journal Devoted to the Study of the Nervous System and Behavior, 63C, 121-131. 
Costantini, M., Ambrosini, E., Scorolli, C., \& Borghi, A. M. (2011). When objects are close to me: affordances in the peripersonal space. Psychonomic Bulletin \& Review, 18(2), 302308.

Culham, J. C., Gallivan, J., Cavina-Pratesi, C., \& Quinlan, D. J. (2008). fMRI investigations of reaching and ego space in human superior parieto-occipital cortex. In R. L. Klatzky, M. Behrmann, \& B. MacWhinney (Eds.), Embodiment, Ego-space and Action (Erlbaum., pp. 247-274). Madwah NJ.

Delevoye-Turrell, Y., Bartolo, A., \& Coello, Y. (2010). Motor representation and the perception of space. In N. Gangopadhyay (Ed.), Perception, Action and Consciousness (pp. 217-242). Oxford: Oxford University Press.

Delorme, A., \& Makeig, S. (2004). EEGLAB: an open source toolbox for analysis of single-trial EEG dynamics including independent component analysis. Journal of Neuroscience Methods, 134(1), 9-21.

Delorme, A., Sejnowski, T., \& Makeig, S. (2007). Enhanced detection of artifacts in EEG data using higher-order statistics and independent component analysis. NeuroImage, 34(4), $1443-1449$.

Fischer, M. H. (2000). Estimating reachability: Whole body engagement or postural stability? Human Movement Science, 19. 297-318

Gabbard, C., Ammar, D., \& Lee, S. (2006). Perceived reachability in single- and multipledegree-of-freedom workspaces. Journal of Motor Behavior, 38(6), 423-9.

Gallese, V. (2007). The "conscious" dorsal stream: embodied simulation and its role in space and action conscious awareness. Psyche, 13, 1-20. 
Gallese, V. (2009). Motor abstraction: a neuroscientific account of how action goals and intentions are mapped and understood. Psychological Research, 73(4), 486-98.

Gallivan, J. P., Cavina-Pratesi, C., \& Culham, J. C. (2009). Is that within reach? fMRI reveals that the human superior parieto-occipital cortex encodes objects reachable by the hand. The Journal of Neuroscience: The Official Journal of the Society for Neuroscience, 29(14), $4381-91$.

Gallivan, J. P., McLean, A., \& Culham, J. C. (2011). Neuroimaging reveals enhanced activation in a reach-selective brain area for objects located within participants' typical hand workspaces. Neuropsychologia, 49(13), 3710-21.

Gastaut, H. J., \& Bert, J. (1954). EEG changes during cinematographic presentation; moving picture activation of the EEG. Electroencephalography and Clinical Neurophysiology, 6(3), 433-44.

Goldman, R. I., Stern, J. M., Engel, J., \& Cohen, M. S. (2002). Simultaneous EEG and fMRI of the alpha rhythm. Neuroreport, 13(18), 2487-92.

Grafton, S. T., \& Hamilton, A. F. de C. (2007). Evidence for a distributed hierarchy of action representation in the brain. Human Movement Science, 26(4), 590-616.

Grèzes, J., \& Decety, J. (2002). Does visual perception of object afford action? Evidence from a neuroimaging study. Neuropsychologia, 40(2), 212-22.

Grusser, O.-J. (1983). Multimodal structure of extrapersonal space. In A. Hein \& M. Jeannerod (Eds.), Spatially oriented behavior (pp. 327-352). New York: Springer-Verlag.

Hall, E. T. (1996). The hidden dimension (Anchor Boo.).

Hari, R. (2006). Action-perception connection and the cortical mu rhythm. Progress in Brain Research, 159, 253-60. 
Heft, H. (1993) A methodological note on overestimates of reaching distance: distinguishing between perceptual and analytical judgments. Ecological psychology, 5, 255-271.

Hediger, H. (1934). Zur Biologie und Psychologie der Flucht bei Tieren. Biologisches Zentralblatt, 54, 21-40.

Holmes, N. P., \& Spence, C. (2004). The body schema and the multisensory representation(s) of peripersonal space. Cognitive Processing, 5(2), 94-105.

Iachini, T., Coello, Y., Frassinetti, F., \& Ruggiero, G. (2014). Body space in social interactions: a comparison of reaching and comfort distance in immersive virtual reality. PloS One, $9(11)$, e111511.

Jasper, H., \& Penfield, W. (1949). Electrocorticograms in man: Effect of voluntary movement upon the electrical activity of the precentral gyrus. Archiv Für Psychiatrie Und Nervenkrankheiten, 183(1-2), 163-174.

Jeannerod, M. (2001). Neural simulation of action: a unifying mechanism for motor cognition. NeuroImage, 14(1 Pt 2), S103-9.

Kleiner, M., Brainard, D., \& Pelli, D. (2007). What's new in Psychtoolbox-3? Perception ECVP Abstract, 36 .

Kuhlman, W. N. (1978). Functional topography of the human mu rhythm. Electroencephalography and Clinical Neurophysiology, 44(1), 83-93.

Lamm, C., Fischer, M. H., \& Decety, J. (2007). Predicting the actions of others taps into one's own somatosensory representations-A functional MRI study. Neuropsychologia, 45, 24802491. 
Llanos, C., Rodriguez, M., Rodriguez-Sabate, C., Morales, I., \& Sabate, M. (2013). Mu-rhythm changes during the planning of motor and motor imagery actions. Neuropsychologia, 51(6), $1019-26$.

Mark, L. S., Nemeth, K., Gardner, D., Dainoff, M. J., Paasche, J., Duffy, M., \& Grandt, K. (1997). Postural dynamics and the preferred critical boundary for visually guided reaching. Journal of Experimental Psychology. Human Perception and Performance, 23(5). 13651379.

Medendorp, W. P., Buchholz, V. N., Van Der Werf, J., \& Leoné, F. T. M. (2011). Parietofrontal circuits in goal-oriented behaviour. The European Journal of Neuroscience, 33(11), 201727.

Muthukumaraswamy, S. D., \& Johnson, B. W. (2004). Primary motor cortex activation during action observation revealed by wavelet analysis of the EEG. Clinical Neurophysiology: Official Journal of the International Federation of Clinical Neurophysiology, 115(8), 17606.

Muthukumaraswamy, S. D., Johnson, B. W., \& McNair, N. a. (2004). Mu rhythm modulation during observation of an object-directed grasp. Brain Research. Cognitive Brain Research, 19(2), 195-201.

Nyström, P., Ljunghammar, T., Rosander, K., \& Hofsten, C. Von. (2011). Using mu rhythm desynchronization to measure mirror neuron activity in infants. Developmental Science, 14(2), 327-35.

Oldfield, R. C. (1971). The assessment and analysis of handedness: the Edinburgh inventory. Neuropsychologia, 9, 97-113. 
Paillard, J. (1991). Motor and representational framing of space. In J. Paillard (Ed.), Brain and space (Oxford Uni., pp. 163-182). Oxford.

Pelli, D. G. (1997). The VideoToolbox software for visual psychophysics: transforming numbers into movies. Spatial Vision, 10(4), 437-42.

Perry, A., \& Bentin, S. (2009). Mirror activity in the human brain while observing hand movements: a comparison between EEG desynchronization in the mu-range and previous fMRI results. Brain Research, 1282, 126-32.

Perry, A., \& Bentin, S. (2011). Motor system contributions to our understanding of others: Evidence from EEG activity in the mu frequency range. Journal of Molecular Neuroscience, 45, S92.

Perry, A., Bentin, S., Bartal, I. B.-A., Lamm, C., \& Decety, J. (2010). "Feeling" the pain of those who are different from us: Modulation of EEG in the mu/alpha range. Cognitive, Affective \& Behavioral Neuroscience, 10(4), 493-504.

Pfurtscheller, G., Brunner, C., Schlögl, A., \& Lopes da Silva, F. H. (2006). Mu rhythm (de)synchronization and EEG single-trial classification of different motor imagery tasks. NeuroImage, 31(1), 153-9.

Pfurtscheller, G., \& Neuper, C. (1994). Event-related synchronization of mu rhythm in the EEG over the cortical hand area in man. Neuroscience Letters, 174(1), 93-96. d

Pineda, J. A. (2005). The functional significance of mu rhythms: translating "seeing" and "hearing" into "doing”. Brain Research. Brain Research Reviews, 50(1), 57-68.

Pineda, J. A., Allison, B. Z., \& Vankov, A. (2000). The effects of self-movement, observation, and imagination on mu rhythms and readiness potentials (RP's): toward a brain-computer 
interface (BCI). IEEE Transactions on Rehabilitation Engineering: A Publication of the IEEE Engineering in Medicine and Biology Society, 8(2), 219-22.

Pineda, J. A., Giromini, L., Porcelli, P., Parolin, L., \& Viglione, D. J. (2011). Mu suppression and human movement responses to the Rorschach test. Neuroreport, 22(5), 223-6.

Previc, F. H. (1998). The neuropsychology of 3-D space. Psychological Bulletin, 124(2), 12364.

Proverbio, A. M. (2012). Tool perception suppresses $10-12 \mathrm{~Hz} \mu$ rhythm of EEG over the somatosensory area. Biological Psychology, 91(1), 1-7.

Quinlan, D. J., \& Culham, J. C. (2007). fMRI reveals a preference for near viewing in the human parieto-occipital cortex. NeuroImage, 36(1), 167-87.

Robinovitch, S. N. (1998). Perception of postural limits during reaching. Journal of Motor Behavior, 30(4), 352-8.

Rochat, P., \& Wraga, M. (1997). An account of the systematic error in judging what is reachable. Journal of Experimental Psychology. Human Perception and Performance, 23(1), 199-212.

Salenius, S., Schnitzler, A., Salmelin, R., Jousmäki, V., \& Hari, R. (1997). Modulation of human cortical rolandic rhythms during natural sensorimotor tasks. NeuroImage, 5(3), 221-8.

Salmelin, R., \& Hari, R. (1994). Spatiotemporal characteristics of sensorimotor neuromagnetic rhythms related to thumb movement. Neuroscience, 60(2), 537-550.

Tallon-Baudry, C., Bertrand, O., Delpuech, C., \& Pernier, J. (1996). Stimulus Specificity of Phase-Locked and Non-Phase-Locked $40 \mathrm{~Hz}$ Visual Responses in Human. J. Neurosci., 16(13), 4240-4249. 
Vishton, P. M., \& Cutting, J. E. (1995). Wayfinding, displacements, and mental maps: velocity fields are not typically used to determine one's aimpoint. Journal of Experimental Psychology. Human Perception and Performance, 21(5), 978-95.

Witt, J. K. (2011). Tool use influences perceived shape and perceived parallelism, which serve as indirect measures of perceived distance. Journal of Experimental Psychology. Human Perception and Performance, 37(4), 1148-56.

Witt, J. K., \& Proffitt, D. R. (2008). Action-specific influences on distance perception: a role for motor simulation. Journal of Experimental Psychology. Human Perception and Performance, 34(6), 1479-92.

Witt, J. K., Proffitt, D. R., \& Epstein, W. (2004). Perceiving distance: a role of effort and intent. Perception, 33(5), 577-90.

Witt, J. K., Proffitt, D. R., \& Epstein, W. (2005). Tool use affects perceived distance, but only when you intend to use it. Journal of Experimental Psychology. Human Perception and Performance, 31(5), 880-8. 


\section{Figure Caption}

Figure 1. (A) Experimental setup composed of the rear screen for stereoscopic projection and showing one seated participant wearing the EEG cap and responding with the pedals. (B) Illustration of a prototypical object (bottle) and (C) the corresponding distorted object resulting from application of the Gaussian scattering algorithm on the prototypical object picture (bottle).

Figure 2: (A) Time Frequency representation of $\mu$ power change $(8 \mathrm{~Hz}-12 \mathrm{~Hz})$ in the centroparietal region (indicated by the 7 electrodes in the scalp map distribution) averaged across participants for the entire time window (from -200 to $1000 \mathrm{~ms}$ ), as a function of the Task (RJ, OI task), Object type (prototypical, distorted) and Space (peripersonal, extrapersonal, boundary of peripersonal space). (B) Mean $\mu$ power change as function of time for the two tasks (RJ, OI task) and Object type (prototypical, distorted). On each plot, blue colour was used for objects presented in peripersonal space, black colour for objects presented at the boundary of peripersonal space and red colour for objects presented in extrapersonal space.

Figure 3: Mean (and SD) $\mu$ power change (in $\%$ relative to baseline) for the Object type (prototypical, distorted) as a function of task (RJ, OI task) and Space (peripersonal, extrapersonal, boundary of peripersonal space).

Figure 4: Upper panel: $\mu$ power change across time in the RJ task for prototypical and distorted objects when presented in the peripersonal or extrapersonal space. Lower panel: variation of statistical p-value associated with the t-test comparison between $\mu$ power change induced by visual presentation of object located in peripersonal versus extrapersonal space as a function of 
time. Horizontal dotted line indicates the significant threshold at $\alpha=.05$ and the vertical line indicates the moment in time when the threshold was reached for the first time (300 ms) in the presence of prototypical objects. 


\section{Appendix}

Complete list of stimuli used.

\begin{tabular}{|c|}
\hline Object \\
\hline Soda bottle \\
\hline Lemonade bottle \\
\hline Beer bottle \\
\hline Soda can \\
\hline Salt cellar \\
\hline Flask \\
\hline Beaker \\
\hline Coffee carafe \\
\hline Jam jar \\
\hline Baby's bottle \\
\hline Toilet cleaner bottle \\
\hline Hourglass \\
\hline Die \\
\hline Aerosol spray can \\
\hline Vase \\
\hline Baseball \\
\hline Tennis ball \\
\hline Dish soap bottle \\
\hline Rubik's cube \\
\hline Roll-on deodorant \\
\hline
\end{tabular}




\section{Highlights:}

- EEG $\mu$ rhythm was analyzed in a reachability judgment \& object identification task

- $\mu$ desynchronization for prototypical objects in peripersonal space in the RJ task

- No $\mu$ desynchronization for distorted objects in both spaces and both tasks

- Motor coding of visual object depends on objects shape and location in space

- Motor coding of visual object depends also on the goal of the perceptual task 


\section{Click here to download high resolution image}

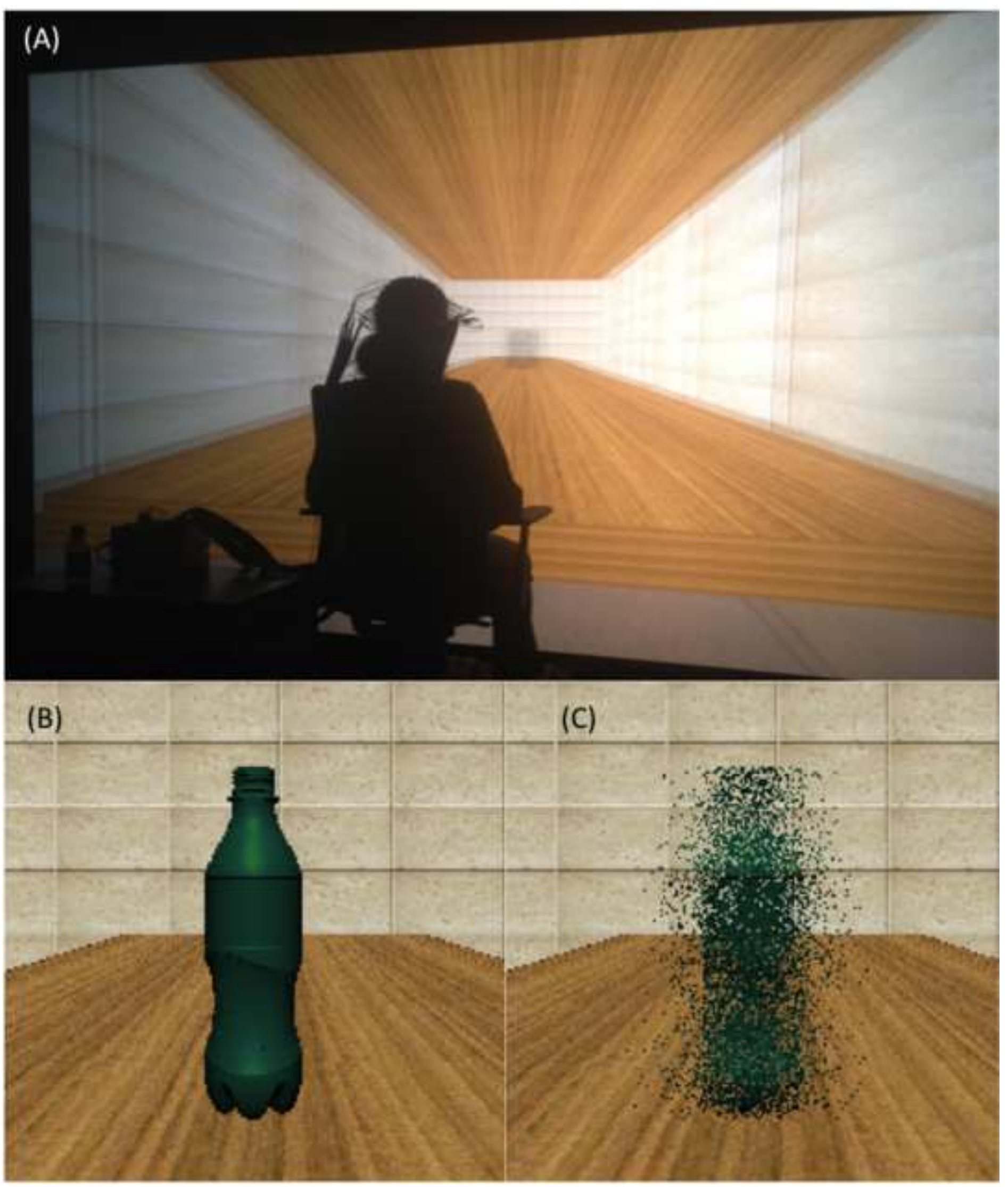


Click here to download high resolution image

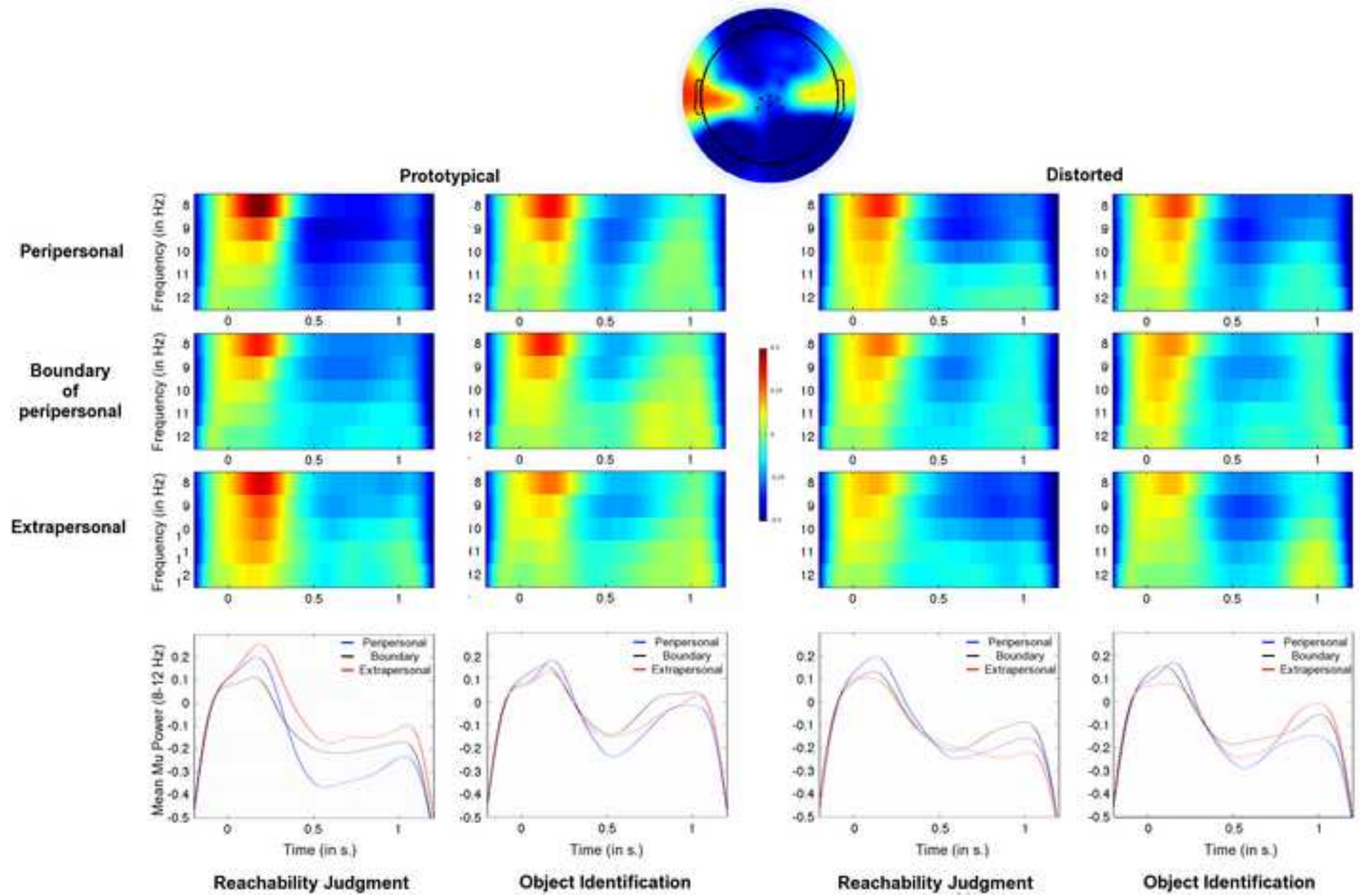




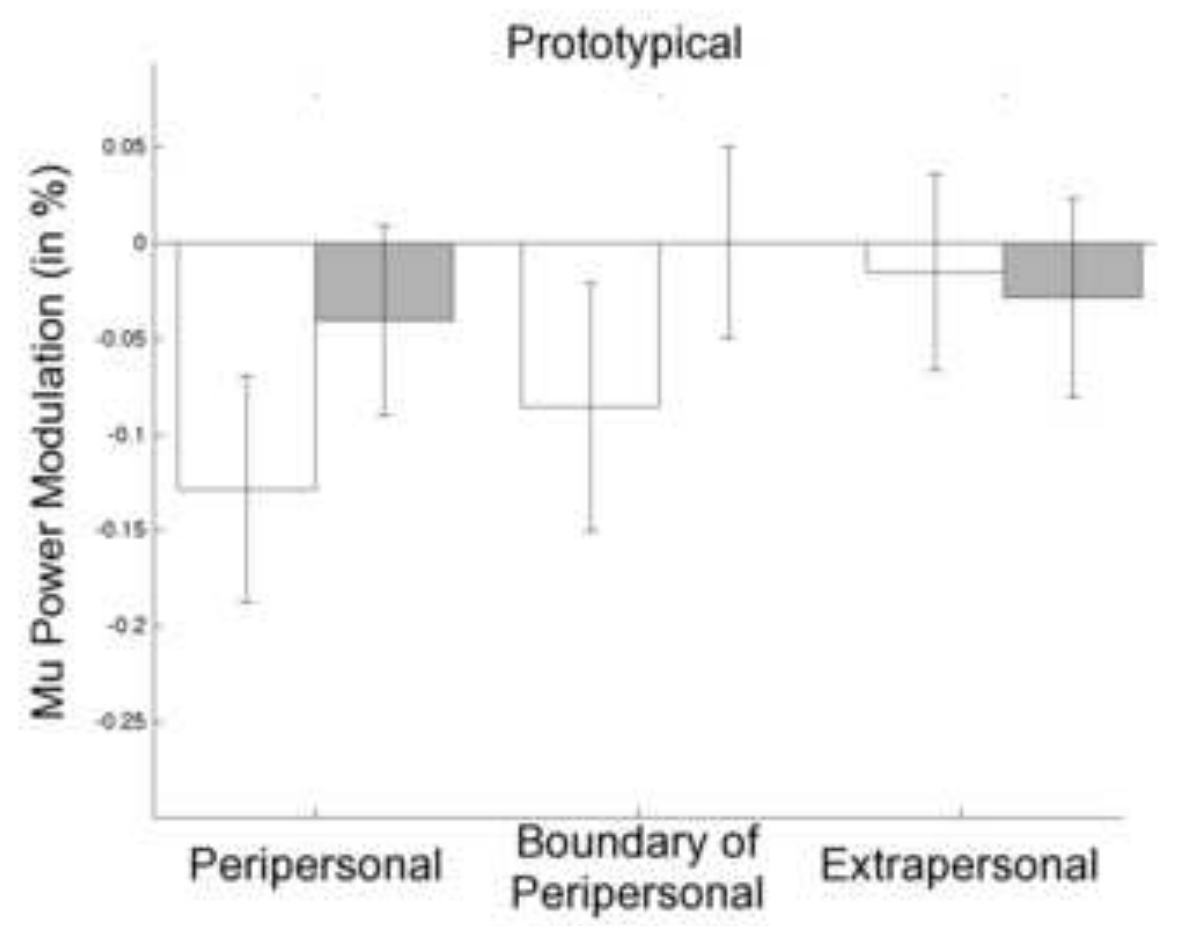

Reachability Judgment

\section{Distorted}

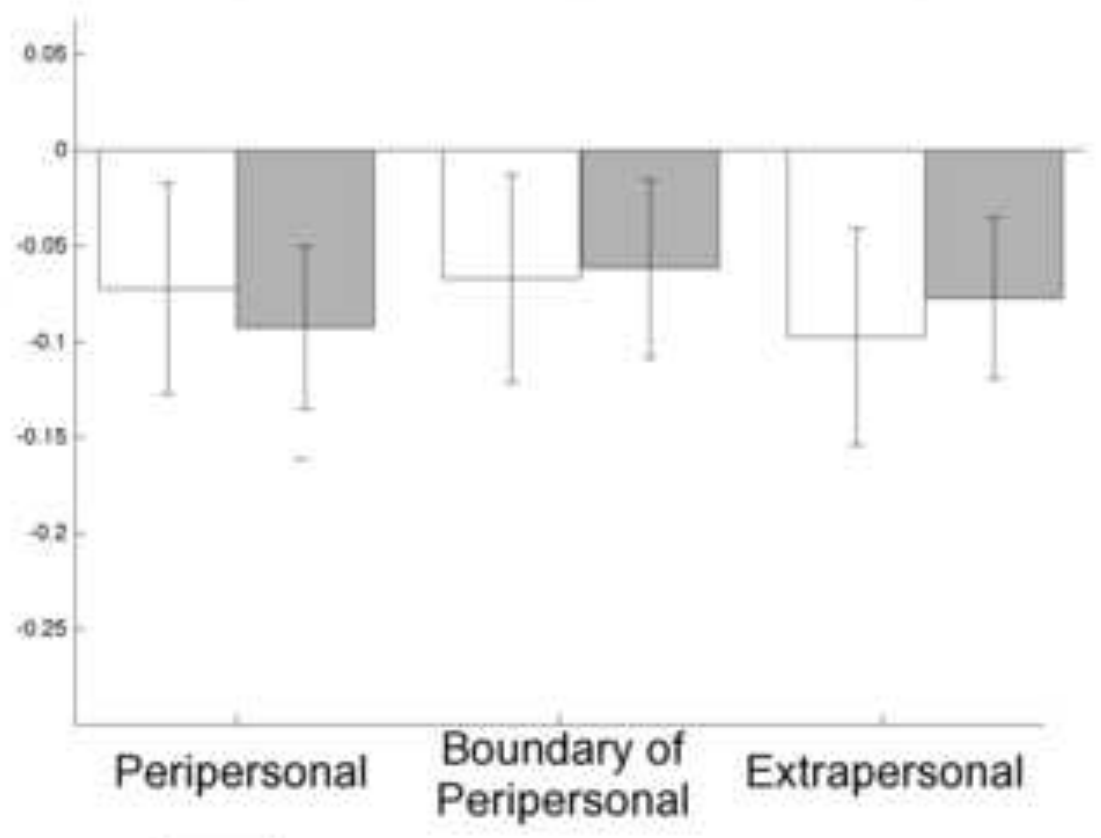

Object Identification 

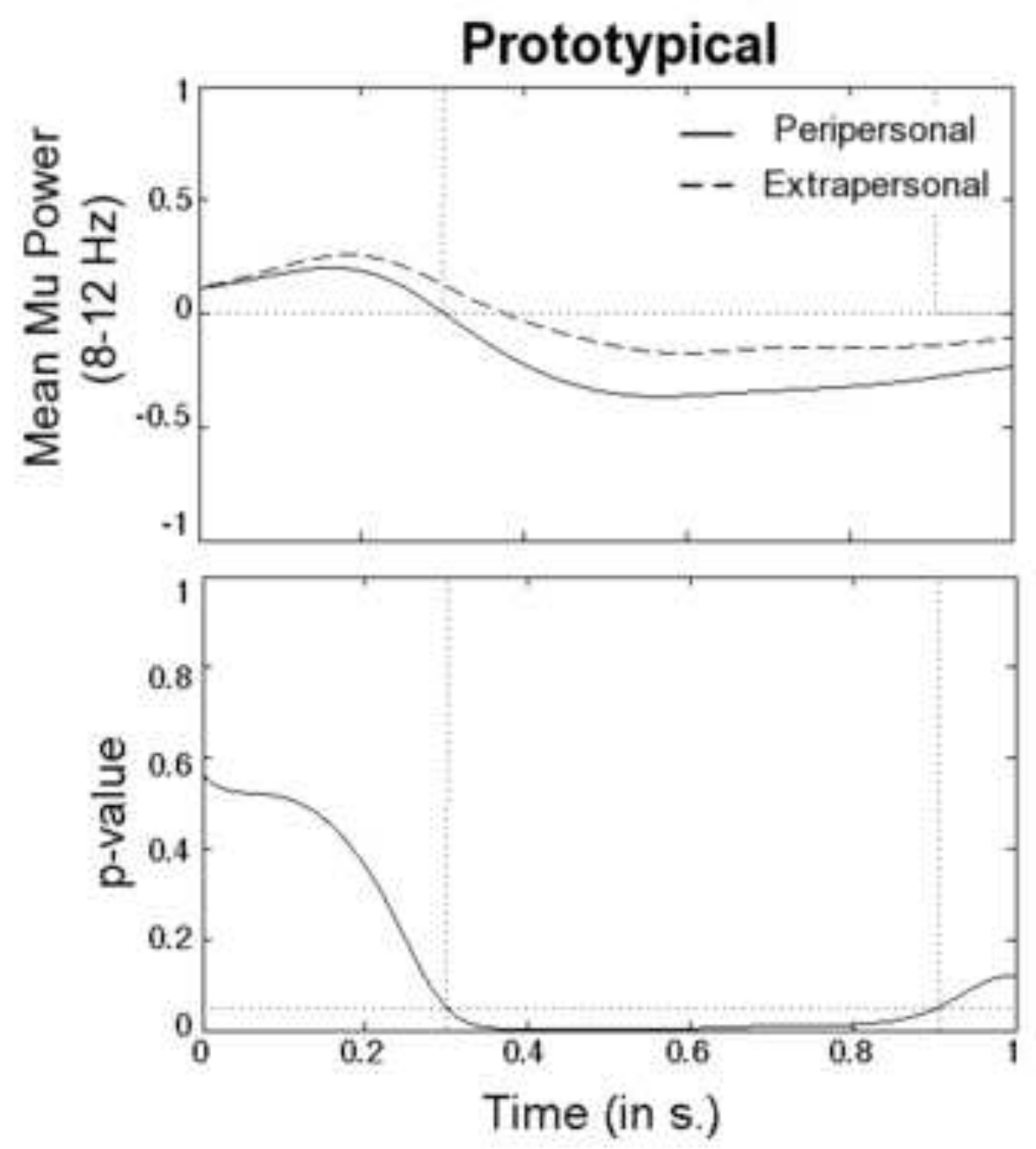

\section{Distorted}
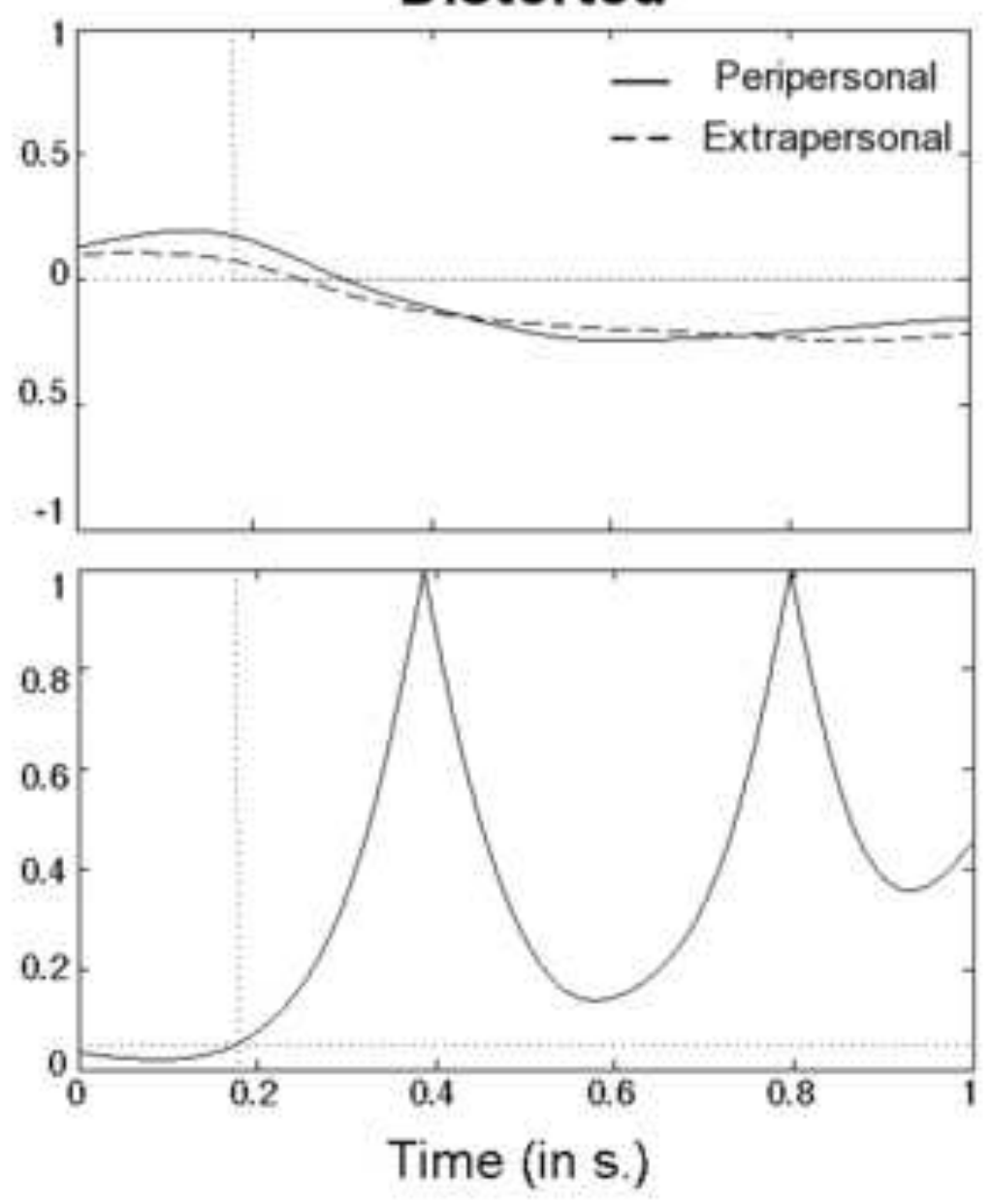